\section{Stressreduktion könnte Fatigue bei SLE lindern}

Azizoddin DR et al. Longitudinal study of fatigue, stress, and depression: role of reduction in stress toward improvement in fatigue. Arthritis Care Res 2020; 72 (10): 1440-1448

Patienten mit systemischem Lupus erythematodes (SLE) leiden sehr häufig an Fatigue, die genauen Ursachen sind unbekannt. Für die Patienten bedeutet die Fatigue eine enorme Einschränkung ihrer Lebensqualität. Eine neue Studie hat untersucht, ob empfundener Stress und Depressionen Prädiktoren für das Auftreten von Fatigue sind.

In der Studie wurden Daten der Lupus-Outcomes-Studie ( $n=650$ Patienten) der University of California San Francisco ausgewertet. Die eingeschlossenen Patienten erfüllten die SLE-Diagnose-Kriterien des American College of Rheumatologists und wurden in strukturierten Telefon-Interviews jährlich u. a. zu Krankheitssymptomen, Medikamenteneinnahme, aber auch nach Fatigue (Short Form 36 (SF-36) vitality scale), Depressionen (Center for epidemiologic studies depression scale CES-D) und empfundenem Stress (4-item-perceived stress scale PS-4) befragt. Die PS-4 fragt u. a. nach dem Gefühl, Kontrolle über das eigene Leben zu haben und in der Lage zu sein, mit seinen Problemen zurecht zu kommen. Alter, Geschlecht, BMI, Ethnizität, Ausbildungsgrad, Einkommen, Krankheitsdauer und Fatigue in der Vorgeschichte waren über die Teilnehmer bekannt.

In der vorliegenden Studie wurden die Daten von 2 aufeinanderfolgenden Interviews ausgewertet. Mithilfe multivariater linearer Regressionsmodelle versuchten die Wissenschaftler Prädiktoren für das Auftreten von Fatigue zu identifizieren. In einem Modell 1 untersuchten sie die Assoziation von Depressionen zum Zeitpunkt 1 (T1) mit Fatigue zum Zeitpunkt 2 (T2); In Modell 2 wurde zusätzlich der zum Zeitpunkt T1 empfundene Stress berücksichtigt, in Modell 3 die Abnahme des Stresses von Zeitpunkt T1 zu T2. Alle Analysen wurden nach Fatigue, Alter, Geschlecht, Fibromyalgie, Schmerzen und SLE-Dauer, Aktivität und Schädigung zum Zeitpunkt T1 kontrolliert.
Das mittlere Alter der Studienteilnehmer betrug 50,6 \pm 12 ,6 Jahre, 92,2\% der Teilnehmer waren Frauen und $68 \%$ waren weiß. Der mittlere SF-36-Fatigue-Score betrug $54,8 \pm 23,9$, der mittlere PS-4 5,3 $\pm 3,6$ und der mittlere CES-D 14,1 $\pm 12,4$.

Die Autoren zeigten in Modell 1, dass eine Depression zu T1 signifikant eine Fatigue zu T2 voraussagt. Wenn allerdings in Modell 2 der empfundene Stress zu T1 berücksichtigt wurde, sagte der Stress ( $\beta=1,7$ [95\% Konfidenzintervall (95\% Cl) 1,1, 2,2]; $\mathrm{P}<0,0001$ ) signifikant Fatigue zu T2 voraus, die Depression war aber kein signifikanter Prädiktor mehr. Eine Stress-Reduktion von T1-zu-T2 war mit einer signifikanten Abnahme von Fatigue assoziiert ( $\beta=-11,8$ [95\% Konfidenzintervall $(95 \% \mathrm{Cl}-15,6,-8,9]$; $\mathrm{P}<0,0001)$. Die Autoren folgern, dass empfundener Stress die Verbindung zwischen Depression und Fatigue darstellt.

FAZIT

Fatigue schränkt die Lebensqualität von Lupus-Patienten erheblich ein. Die vorliegende Studie zeigt, dass Stress das Auftreten von Fatigue bei Lupus beeinflusst und eine Stressreduktion Fatigue verbessern könnte. Lupus-Patienten, so argumentieren die Autoren, könnten gezielt nach empfundenem Stress befragt werden, um möglichst früh Interventionen zur Stressreduktion anbieten zu können. Dies könnte die Lebensqualität der Patienten verbessern.

Marisa Kurz M. Sc. B. A. München 